\title{
Risk Factors for Scrub Typhus, Murine Typhus, and Spotted Fever Seropositivity in Urban Areas, Rural Plains, and Peri-Forest Hill Villages in South India: A Cross-Sectional Study
}

\author{
Carol S. Devamani, ${ }^{1}$ Wolf-Peter Schmidt, ${ }^{2}$ Koya Ariyoshi, ${ }^{3}$ Arumugam Anitha, ${ }^{4}$ Saravanan Kalaimani, ${ }^{4}$ and John A. J. Prakash ${ }^{4 *}$ \\ ${ }^{1}$ Department of RUHSA, Christian Medical College, Vellore, India; ${ }^{2}$ Department for Disease Control, London School of Hygiene and Tropical \\ Medicine, London, United Kingdom; ${ }^{3}$ Department of Clinical Medicine, Institute of Tropical Medicine, Nagasaki University, Nagasaki, Japan; \\ ${ }^{4}$ Department of Clinical Microbiology, Christian Medical College, Vellore, India
}

\begin{abstract}
Scrub typhus and spotted fever group rickettsioses are thought to be common causes of febrile illness in India, whereas they rarely test for murine typhus. This cross-sectional study explored the risk factors associated with scrub typhus, tick-borne spotted fever, and murine typhus seropositivity in three different geographical settings, urban, rural, and hill villages in Tamil Nadu, South India. We enrolled 1,353 participants living in 48 clusters. The study included a questionnaire survey and blood sampling. Blood was tested for Orientia tsutsugamushi (scrub typhus), Rickettsia typhi (murine typhus), and spotted fever group Rickettsia IgG using ELISA. The seroprevalence of scrub typhus, spotted fever, and murine typhus were $20.4 \%, 10.4 \%$, and $5.4 \%$, respectively. Scrub typhus had the highest prevalence in rural areas $(28.1 \%)$, and spotted fever was most common in peri-forested areas (14.9\%). Murine typhus was more common in rural $(8.7 \%)$ than urban areas (5.4\%) and absent in peri-forested hill areas. Agricultural workers had a higher relative risk for scrub typhus, especially in urban areas. For murine typhus, proximity to a waterbody and owning a dog were found to be major risk factors. The main risk factors for spotted fever were agricultural work and living in proximity to a forest. Urban, rural plains, and hill settings display distinct epidemiological pattern of Orientia and rickettsial infections. Although scrub typhus and spotted fever were associated with known risk factors in this study, the findings suggest a different ecology of murine typhus transmission compared with other studies conducted in Asia.
\end{abstract}

\section{INTRODUCTION}

Scrub typhus is a febrile illness caused by Orientia tsutsugamushi, bacteria related but not identical to the genus Rickettsia (both are part of the family Rickettsiaceae). ${ }^{1}$ The true rickettsial infections include among others the highly diverse spotted fever group rickettsioses (SFGR) and murine typhus (Rickettsia typhi). Scrub typhus is transmitted by the larvae (chiggers) of trombiculid mites which infect mammals as incidental hosts. ${ }^{2}$ Spotted fever is largely transmitted by hard ticks, ${ }^{3}$ whereas murine typhus is transmitted by contact with fleas. ${ }^{4,5}$ Scrub typhus occurs over much of tropical and subtropical East Asia, South Asia, and Southeast Asia. ${ }^{6}$ The disease has recently been identified in Chile ${ }^{7}$ and possibly East Africa. ${ }^{8}$ Spotted fever group rickettsioses and murine typhus occur worldwide.

Scrub typhus and SFGR are thought to be common causes of febrile illness in India. ${ }^{9-11}$ Serological evidence also exists for murine typhus, but is rarely tested for. Delays in disease recognition and treatment may cause a considerable number of preventable deaths across India. ${ }^{2,9}$

This study explored risk factors associated with scrub typhus, spotted fever, and murine typhus seropositivity in three different geographical settings (urban, rural plains, and periforested hills) in Tamil Nadu, South India.

\section{METHODS}

Study design. The study was a cross-sectional survey, conducted in Vellore, Thiruvannamalai, and Salem districts, all in Tamil Nadu (India). These districts are characterized by three broad geographic areas: urban, rural plains, and rural hill areas up to about 1,000 m elevation. In rural areas (plains and

${ }^{*}$ Address correspondence to John A. J. Prakash, Department of Clinical Microbiology, Christian Medical College, Ida Scudder Road, Vellore 632004, India. E-mail: prakjaj@cmcvellore.ac.in hills), the major crops are rice, sugarcane, sorghum, coconut, pulses, mango, and turmeric. Animal husbandry mainly includes cattle, goats, and poultry. In hill areas, most villages lie at the edge of mountain forests at varying degrees of degradation (peri-forest). In the rural plains, there are isolated patches of secondary forest/dense bush and commercial plantations (e.g., teak and gum tree). The climate is tropical savannah. Monsoon rains occur between June and December. The scrub typhus season approximately lasts from August to February, with a peak from November to January. Scrub typhus occurs sporadically between seasons. ${ }^{12}$ The seasonality of spotted fever and murine typhus has not been studied. The study was conducted in all three geographic settings (urban communities, rural plain villages, and periforest hill villages). The study consisted of a questionnaire survey and a blood sample from one individual per enrolled household. Figure 1 shows the areas surveyed.

Enrollment of communities, households, and participants. The selection of villages aimed at enrolling high-risk clusters of Orientia/rickettsial infection. We did not aim at enrolling a representative sample of the total population of the three districts, as this may have reduced the power of the study to detect risk factors. Selection of study participants was performed in two stages. In the first stage, villages (rural plains and peri-forest hill areas) and urban communities were selected. First, we compiled a list of all communities and villages in the districts with cases of scrub typhus or rickettsial infections admitted to the Christian Medical Collage (CMC), Vellore, in the 5 years before the study. A village or urban community was eligible for enrollment if at least two cases of scrub typhus or rickettsial infection had been admitted during that time. This list comprised 55 urban communities and 53 rural plain villages from two districts (Vellore and Thiruvannamalai) and was dominated by scrub typhus, as the causative agent of more than $90 \%$ of all hospitalized Orientia/rickettsial infections. For the peri-forest hill villages, this approach had to be modified. Cases occurring in these 


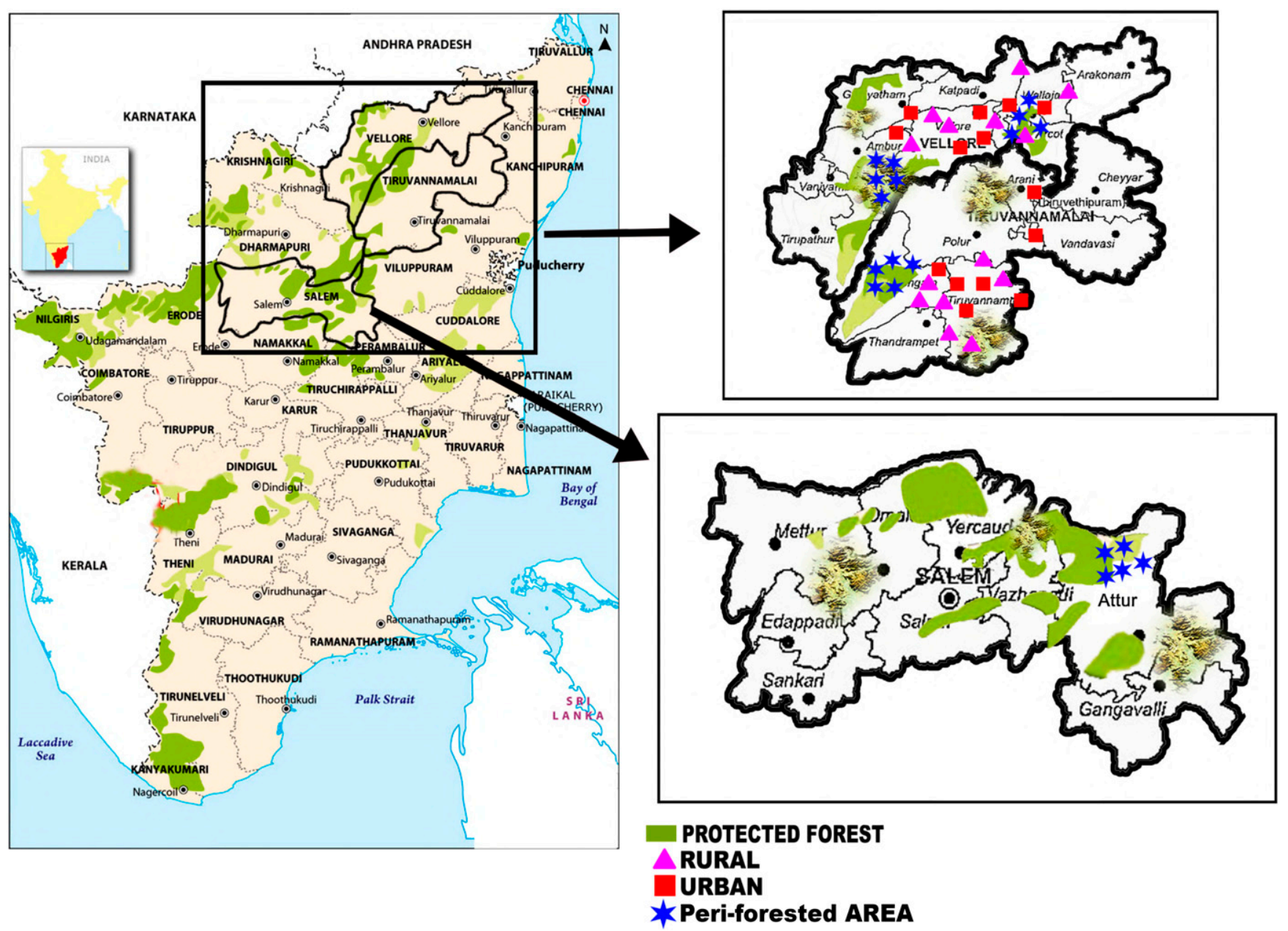

FigURE 1. Study area. This figure appears in color at www.ajtmh.org.

areas are often treated locally or at government hospitals, presumably because of difficulties in accessing health care at tertiary care centers due to transport problems or financial constraints. Because of these limitations in our knowledge on case numbers at the village level, we chose villages in hill areas where scrub typhus and rickettsial infections were thought to occur based on the experience of local doctors. Within these hill areas of known or presumed endemicity for scrub typhus and rickettsial infections, we purposively selected villages that were easily accessible by road, and added villages from a third district (Salem).

We sampled 30 households per community/village in urban communities and rural plain villages, and 25 households per village in hill areas. Within a chosen community or village, enrollment of households was performed by systematic sampling of every nth household starting from the center of a village with the direction of the walk determined by a flip of a coin (villages situated along a road) or the spin of a bottle (villages/urban communities consisting of clustered houses). The sampling interval was determined by the size of the community/village based on available census data, divided by the required number of participants (30 in urban communities/ rural plain villages and 25 in hill villages). The resulting number was rounded down to the next integer. If staff reached the boundary of a village or cluster, they went back to the starting point and chose the opposite direction.
Within a household, one participant was chosen at random from the household members present at the time of the visit. Because of logistical constraints, households without anyone present were not revisited.

Questionnaire survey and blood sampling. We administered a questionnaire survey to the selected participants covering sociodemographic, socioeconomic, occupational, geographic, and water/sanitation data. The questionnaire included observation of the household environment and information on livestock and pet animals. The distance to the nearest lake, pond, and forest was estimated using satellite imaging (google maps), with assistance of local residents. A 4- $\mathrm{mL}$ venous blood sample, collected in a serum tube, was taken from each participant. The blood was stored in a cool box and transported to the laboratory (CMC, Vellore) either on the same day or within 3 days of collection (Salem district).

Laboratory analysis. After collection, blood samples were brought to CMC. Serum was separated from the clot and stored at $-70^{\circ} \mathrm{C}$ until testing. Samples not transported on the same day to $\mathrm{CMC}$ were kept in a refrigerator at $-4^{\circ} \mathrm{C}$ after centrifuging the sample to separate serum. IgG antibodies to O. tsutsugamushi (56 kDa antigens from Karp, Kato, Gilliam, and TA716 strains) were detected in serum using the scrub typhus IgG ELISA system (InBios International Inc., Seattle, WA). For murine typhus IgG detection, we used the $R$. typhi IgG ELISA (Fuller Laboratory, Fullerton, CA) which covers the 
species-specific protein rOmp B. For spotted fever lgG, we used the Rickettsia conorii ELISA IgG/lgM (Vircell, Granada, Spain).

The commercially available ELISA tests used in this study do not come with cutoff points for the optical density (OD) to define seropositivity that are generally agreed on or recommended by the manufacturers for use in cross-sectional surveys. To define seropositivity, we used an OD cutoff of 1.5 for all three infections. This was based on earlier studies in Vellore district that demonstrated a marked bimodal distribution for scrub typhus IgG in the area. ${ }^{13,14}$ A similar bimodal pattern was found in this study (Figure 2A). The value of 1.5 was chosen as the approximate low point between the two peaks.
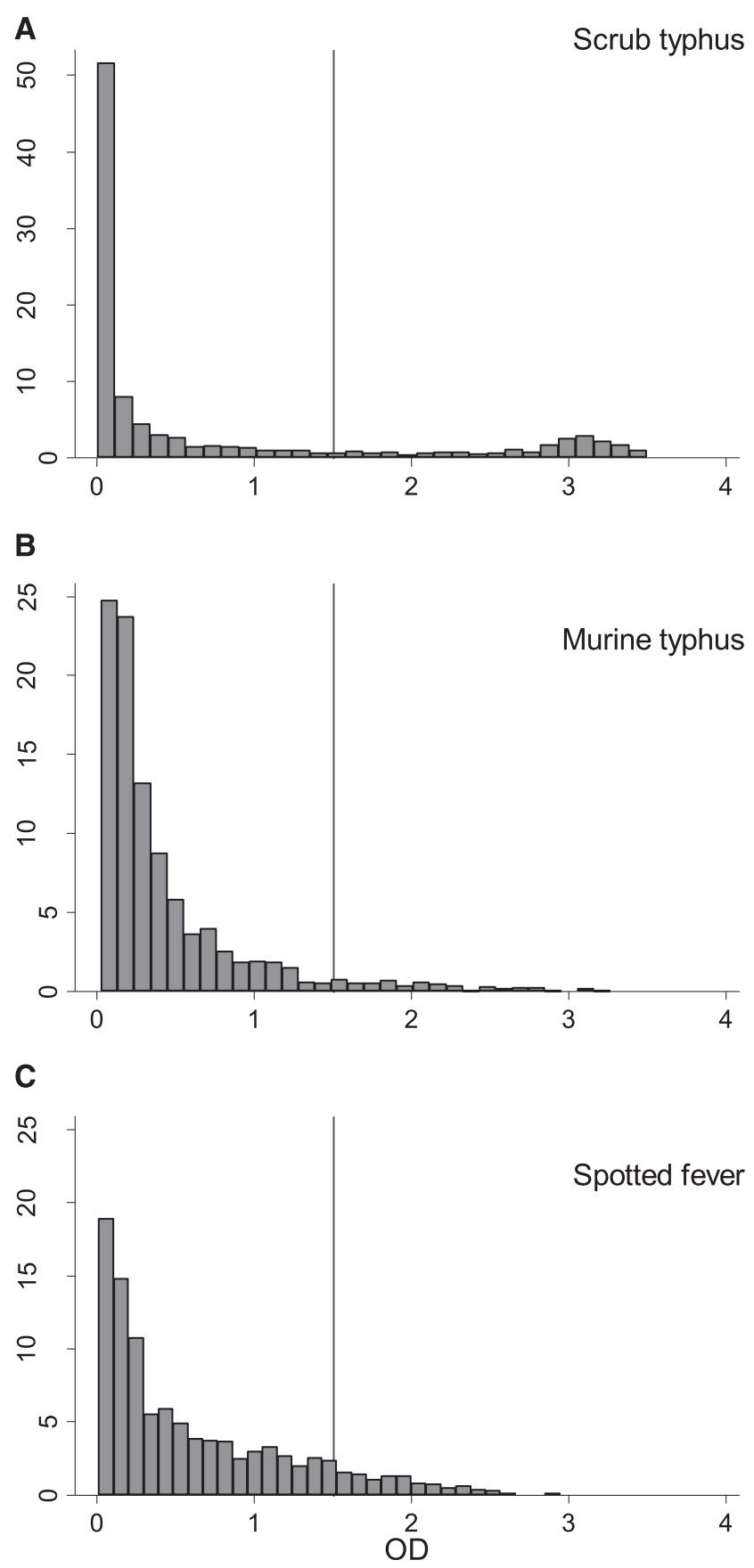

FIGURE 2. ELISA optical densities.
For murine typhus and spotted fever, there was no clear bimodal pattern in the OD (Figure $2 \mathrm{~B}$ and $\mathrm{C}$ ), which made defining a cutoff difficult. In the absence of data on the ELISA OD of IgG antibodies for murine typhus and spotted fever following infection over time, we pragmatically chose the same cutoff as for scrub typhus for both infections (OD = 1.5).

Sample size. For each geographic stratum (urban/rural/hill), we aimed at determining lgG seroprevalence with a margin of error of $5 \%$. Assuming a seroprevalence of $20 \%$ resulted in a crude sample size of 246 people per stratum. We applied a design effect of 2 , resulting in an intended sample size of 492 per stratum.

Statistical analysis. All analyses were carried out in STATA 14 (StataCorp LLC, College Station, TX). The primary outcome for the study was seropositivity for scrub typhus, murine typhus, and spotted fever. Prevalence estimates and Cls were calculated using the STATA svy: proportion command with logit Cls. Coinfection with two pathogens was defined as an individual being lgG seropositive for both pathogens.

Prevalence risk ratios were calculated using Poisson regression. Cls were adjusted for the binomial distribution of the data and clustering at the community/village level using robust standard errors. $P$-values for interaction terms were calculated using likelihood ratio tests. Multivariable analysis was carried out including variables that were associated with the outcomes based on effect size and biological plausibility. Intra-class correlation coefficients (ICCs) for clustering of seroprevalence at the community/village level were calculated using the loneway command in STATA.

Ethics. The study was approved by CMC's Institutional Review Board, IRB no. 9369. Written consent was obtained from all adult participants. Written or verbal assent was obtained from minors, alongside written consent from their parents/guardians.

\section{RESULTS}

We enrolled 1,353 participants from 16 urban communities, 17 rural plain villages, and 15 peri-forest hill villages (Table 1). Of these, $63 \%$ were female and $71 \%$ were older than 30 years. Using an ELISA cutoff of 1.5 OD for all pathogens, the overall seroprevalence was highest for scrub typhus, followed by spotted fever and murine typhus (Table 1). Scrub typhus had the highest seroprevalence of all pathogens in urban areas and rural plains. Spotted fever had a higher seroprevalence in hill villages than in urban and rural plain areas. No participant from hill villages was seropositive for murine typhus (Table 1).

There was some evidence that pairwise dual seropositivity among any of the three pathogens was more common than expected by chance. Dual seropositivity of scrub typhus and spotted fever affected 3\% of individuals ( $n=40$, versus 28.8 expected by chance). Dual seropositivity of scrub typhus and murine typhus was observed in $1.9 \%$ of participants $(n=26$, versus 14.9 expected). Murine typhus/spotted fever dual seropositivity was very rare, affecting only $0.9 \%$ of participants ( $n=12$, versus 7.6 expected).

In univariable analysis, females were more commonly seropositive for scrub typhus and murine typhus than males. For spotted fever, there was no difference. Risk factors markedly associated with scrub typhus seropositivity in univariable analysis included older age, lower education 
TABLE 1

Univariable analysis of risk factors

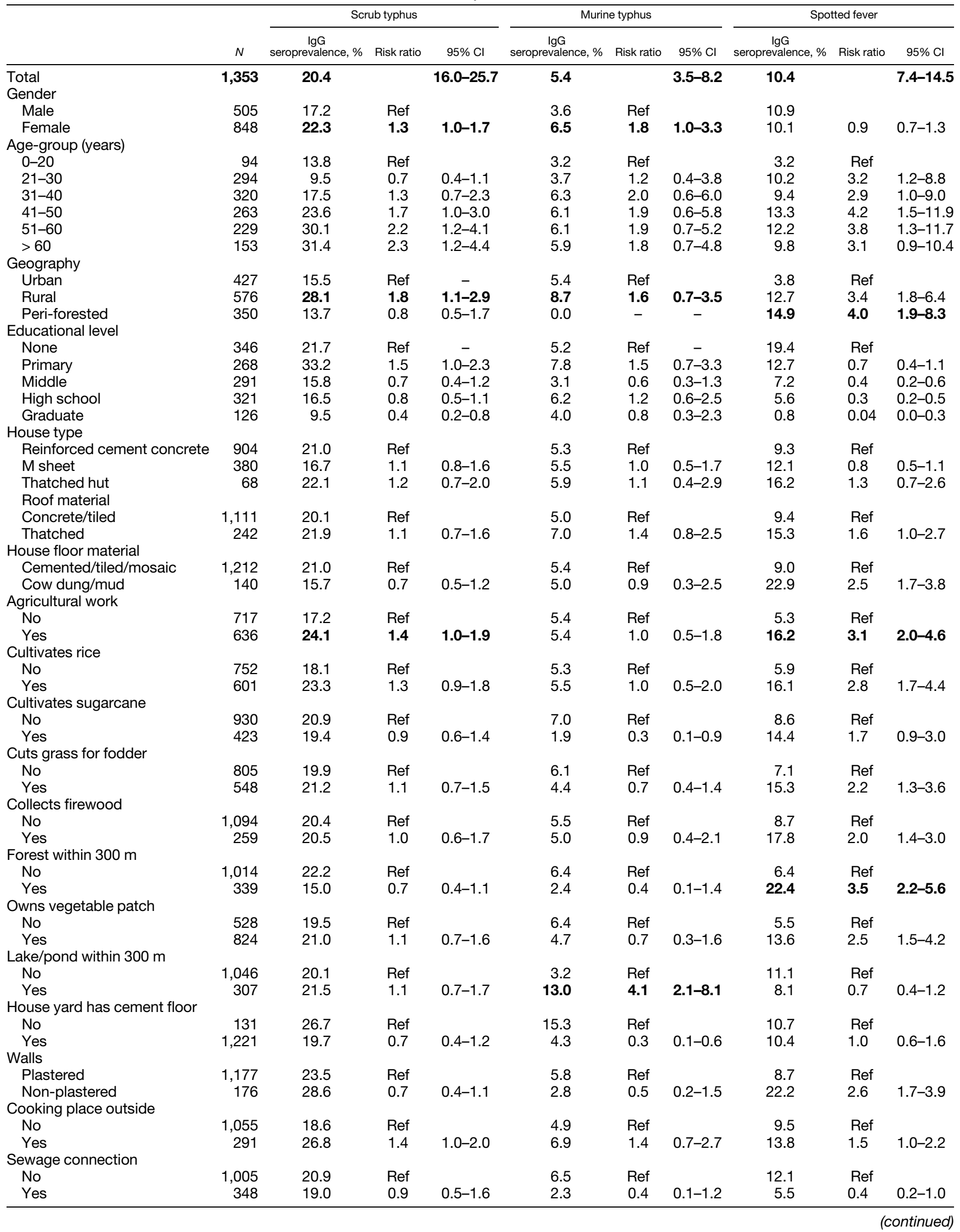




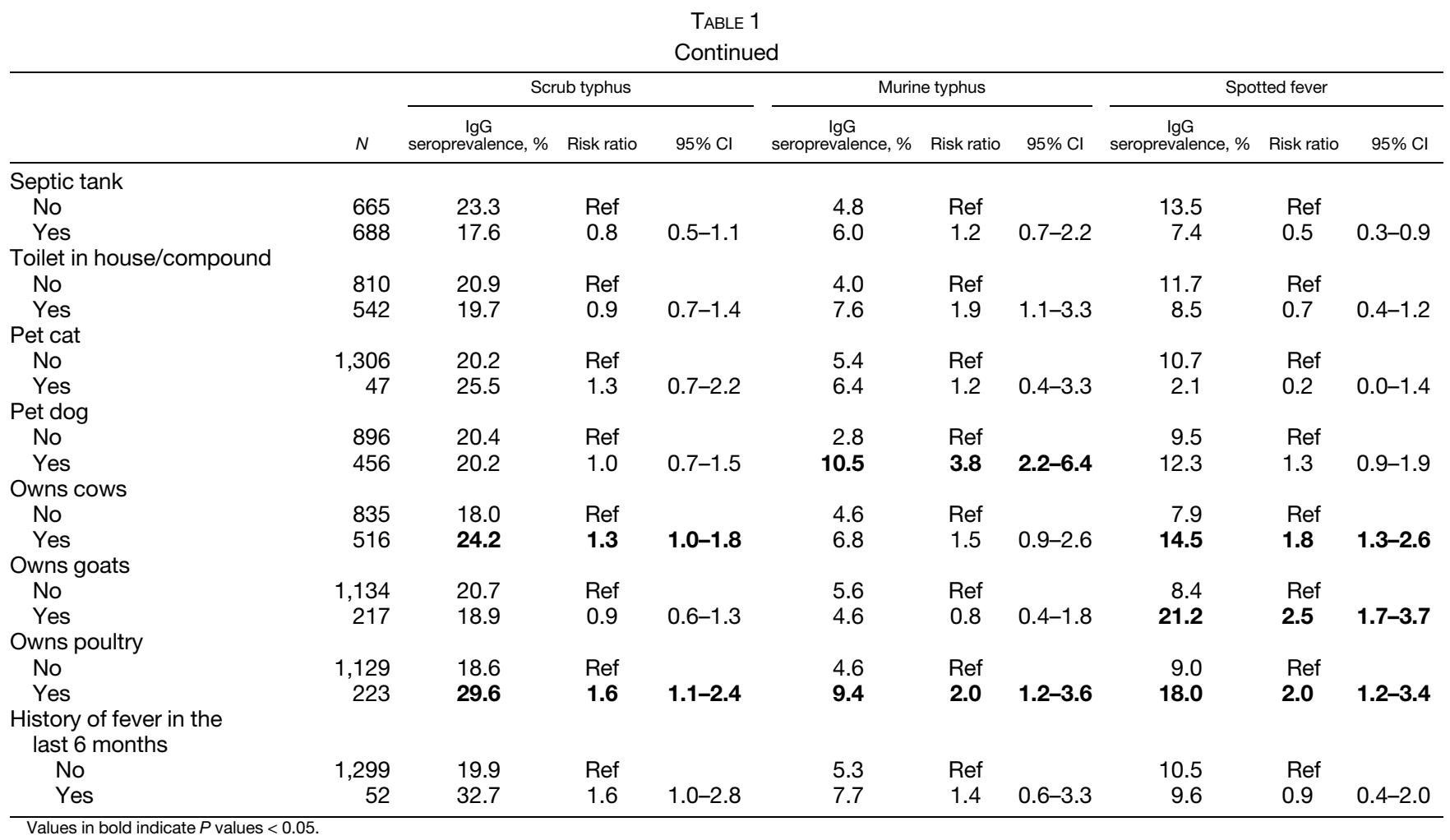

level, agricultural work, an outside cooking place, cow ownership, poultry ownership, and a history of fever in the last 6 months.

Risk factors markedly associated with murine typhus seropositivity in univariable analysis included absence of a forest within $1 \mathrm{~km}$, an uncemented house yard, and dog and poultry ownership. Seroprevalence appeared to be particularly high for participants living within $300 \mathrm{~m}$ of a pond or lake (Figure 3A). For this graph, peri-forest hill areas were excluded as the prevalence of murine typhus was zero.

In univariable analysis, there was a marked association between spotted fever seropositivity and lower education level. Other factors associated with spotted fever were thatched house roof, mud house floor, agricultural work, grass cutting for fodder, firewood collection, proximity to forest, ownership of a vegetable patch, unplastered walls, outside cooking, and ownerships of cows, goats, and poultry. Figure $3 \mathrm{~B}$ shows the association between distance to forest and spotted fever seroprevalence, confirming proximity to a forest as a strong risk factor.

In further stratified analysis, we explored whether there was effect modification of these risk factors by geography (urban/ rural/hill). The association between farming practices (agriculture, and cow and poultry ownership) and scrub typhus was much stronger in urban areas than in rural and hill villages where seroprevalence was overall higher (Table 2). For agricultural work, the test for interaction (urban versus rural + hill) showed a $P$-value of $<0.001$. For murine typhus, the risk ratios for pet dog ownership and proximity to lake/pond were somewhat stronger in urban than in rural areas (Table 3, test for interaction $P=0.36$ and $P=0.39$ ). For spotted fever (Table 4 ), the association with cutting grass was somewhat stronger in hill areas (test for interaction $P=0.34$ ).
In multivariable analysis (Table 5), the following variables were included: gender, age, geography, education, agricultural work, owning a cow, owning poultry, owning goat, owning pet dog, collecting grass, collecting firewood, own vegetable patch, forest within $300 \mathrm{~m}$, lake/pond within $300 \mathrm{~m}$, and house yard with cement floor. Older age and agricultural work remained markedly associated with scrub typhus. There was some evidence that owning a pet dog and proximity to lake or pond remained associated with murine typhus (approximately doubling the prevalence risk). Level of education remained a strong risk factor for spotted fever, alongside agricultural work and to a lesser degree owning a goat. Cutting grass was no longer associated with spotted fever after adjustment in multivariable analysis. The ICC for clustering at the village/community level was 0.14 for scrub typhus, 0.08 for murine typhus (excluding hill villages where the prevalence was zero), and 0.11 for spotted fever. Values mentioned in bold in Tables 1 and 5 are those found significant by univariable and multivariable analysis.

\section{DISCUSSION}

This cross-sectional study explored the seroprevalence of scrub typhus, spotted fever, and murine typhus, and associated risk factors. Scrub typhus had the highest prevalence in rural areas, whereas spotted fever was most common in periforested areas. Murine typhus was more common in rural than in urban areas and absent in peri-forested areas.

Agricultural workers had a higher risk for scrub typhus, especially in urban areas where the overall risk was lower. Older age was also found to be a risk factor for scrub typhus, but not for murine typhus and spotted fever. For murine typhus, proximity to a lake/pond was found to be a major risk factor 

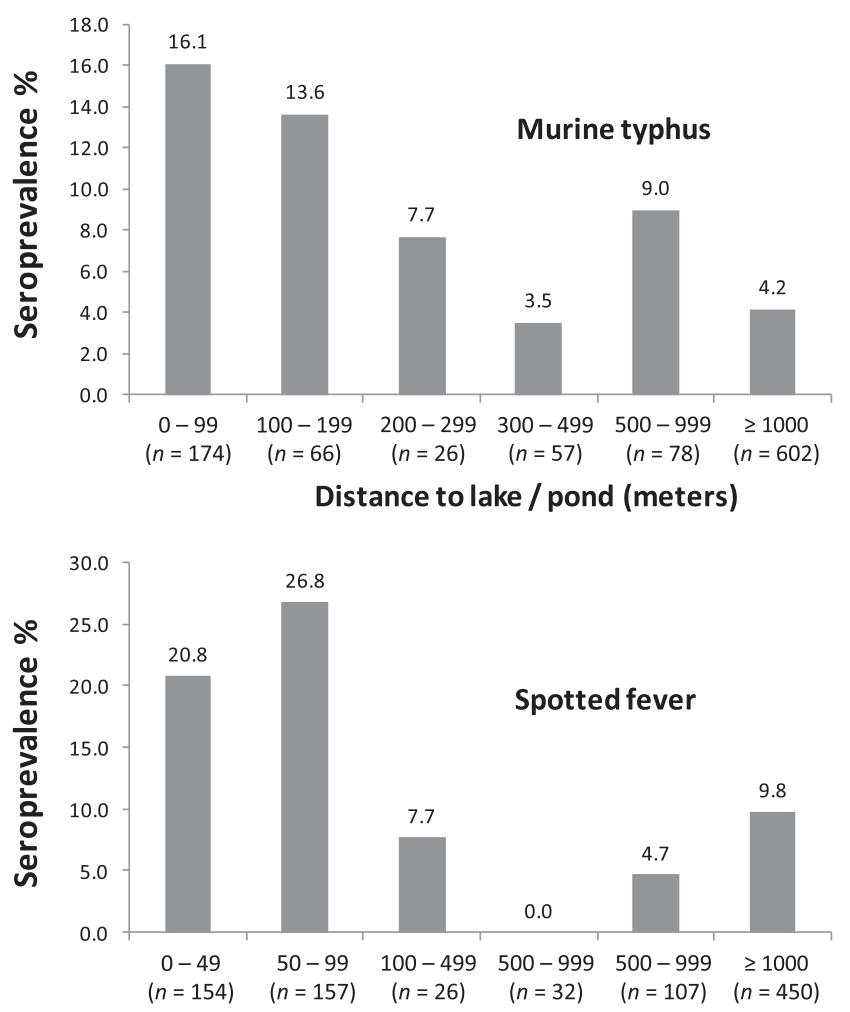

Distance to forest (meters)

FIGURE 3. (A) Association between distance to the nearest lake or pond and murine typhus seroprevalence (excluding hill villages). (B) Association between distance to forest and spotted fever seroprevalence (excluding urban areas).

along with owning a dog. The main risk factors for spotted fever were agricultural work and proximity to a forest.

In line with these findings, studies conducted in Laos, Korea, Indonesia, China, and Malaysia identified female gender, older age, and farming work as risk factors, with the infection being generally more common in rural areas., ${ }^{5,15-22}$ Urban areas have, however, long been known to harbor scrub typhus in endemic countries. ${ }^{23}$ Rickettsioses other than scrub typhus are also not uncommon in urban areas, as reported by Tshokey and others ${ }^{24}$.

A prospective case-control study by George and others ${ }^{11}$ conducted in 2013 showed that farming activities were a risk factor for acquiring scrub typhus in adults. In further studies, the risk of scrub typhus was associated with being female, age $>60$ years, ${ }^{13}$ in agricultural laborers, being bare-chested at home, and living in dwellings adjacent to scrub land. ${ }^{12} \mathrm{~A}$ pediatric case-control study suggested scrub typhus is more likely in children who have pets and stay in houses less than $100 \mathrm{~m}$ from a waterbody and bushes within $5 \mathrm{~m} .{ }^{25}$ The strong association of agricultural work with scrub typhus in urban areas in our study suggests that infestation with mites may often take place away from urban areas, for example, in those who own agricultural land and go to rural areas for cultivation.

In urban Laos, proximity to market places and dense urban communities were risk factors. ${ }^{15}$ However, in our setting, murine typhus was found to be more common in rural areas with possible risk factors including proximity to waterbodies and ownership of a dog, both of which are common in rural settings. Although fleas transmitting murine typhus have classically been shown to be carried by cats, rats, and opossums ${ }^{26}$ studies have also shown dogs to carry cat fleas (Ctenocephalides felis) and to be infected with R. typhi. ${ }^{4,27}$ The association with proximity to waterbodies may, however, also suggest a role of rodents in maintaining transmission. A similar association with waterbodies has been found for leptospirosis, for which rodents are implicated in transmission. ${ }^{28}$ Therefore, our findings suggest that the possible ecology of murine typhus is different in our setting as compared with others areas in Asia such as Laos. ${ }^{15}$

Little is known about the occurrence of tick-borne SFGR in India. ${ }^{3}$ Only a few case series have been published. ${ }^{10,29-32}$ In our study, spotted fever was found to be common among those living in proximity to a forest and agricultural workers, which is in line with studies conducted in Malaysia, Thailand, Indonesia, and Brazil. ${ }^{33-41}$

The study is mainly limited by the pragmatic choices for ELISA cutoff points, the possibility of cross-reactivity among the three infections, and the approach to enroll participants. Seroprevalence estimates depend on the chosen cutoff point for the OD. Furthermore, the interpretation of seropositivity in an individual depends on the average duration an individual remains seropositive after infection. For example, if infected individuals remain seropositive for life, then seropositivity reflects the cumulative lifetime risk of asymptomatic (serological) or symptomatic infection. However, IgG positivity may only persist for a limited amount of time. A study in Vellore district suggested that most people may revert to seronegativity within 3 years after scrub typhus infection. ${ }^{42}$ However, the bimodal distribution of OD values and the near-linear increase in seropositivity with age may suggest that scrub typhus IgG may persist for many years after infection, at least in a sizable proportion of infected. ${ }^{14}$ Similar data are not available for spotted fever and murine typhus. This lack of data on IgG response following infection makes the choice for a suitable OD cutoff and the interpretation of seroprevalence estimates difficult, in particular for murine typhus and spotted fever. In this study, seroprevalence may mean little more than the proportion of the population that has been infected within months or years before the survey. These uncertainties in defining a valid cutoff and in the duration of IgG antibody persistence need to be borne in mind when interpreting absolute prevalence estimates. They should, however, play a lesser role when calculating relative effect measures such as the prevalence risk ratio for different risk factors.

Cross-reactivity among Orientia and rickettsial antibodies has been mainly studied in ELISA tests for acute infection (IgM). ${ }^{43}$ Data on cross-reactivity of $\lg G$ antibodies are limited. Murine typhus and spotted fever group rickettsiosis antibodies may be particularly prone to cross-react with scrub typhus antigens as these are caused by related organisms. A study from an urban setting in Laos jointly examined the prevalence of positive scrub typhus and murine typhus $\lg G$ antibodies (based on ELISA tests) in the general population, demonstrating different geographic risk factors for the two infections. ${ }^{15}$ In this study of 2002 people, 314 were found to be positive only for scrub typhus antibodies, 360 positive only for murine typhus antibodies, whereas 80 were positive for both antibodies. The expected number of dual positives assuming independence between the two infections would have been 86 cases-very similar to the observed 80 . If cross-reactivity 
TABLE 2

Scrub typhus effect modification by geography

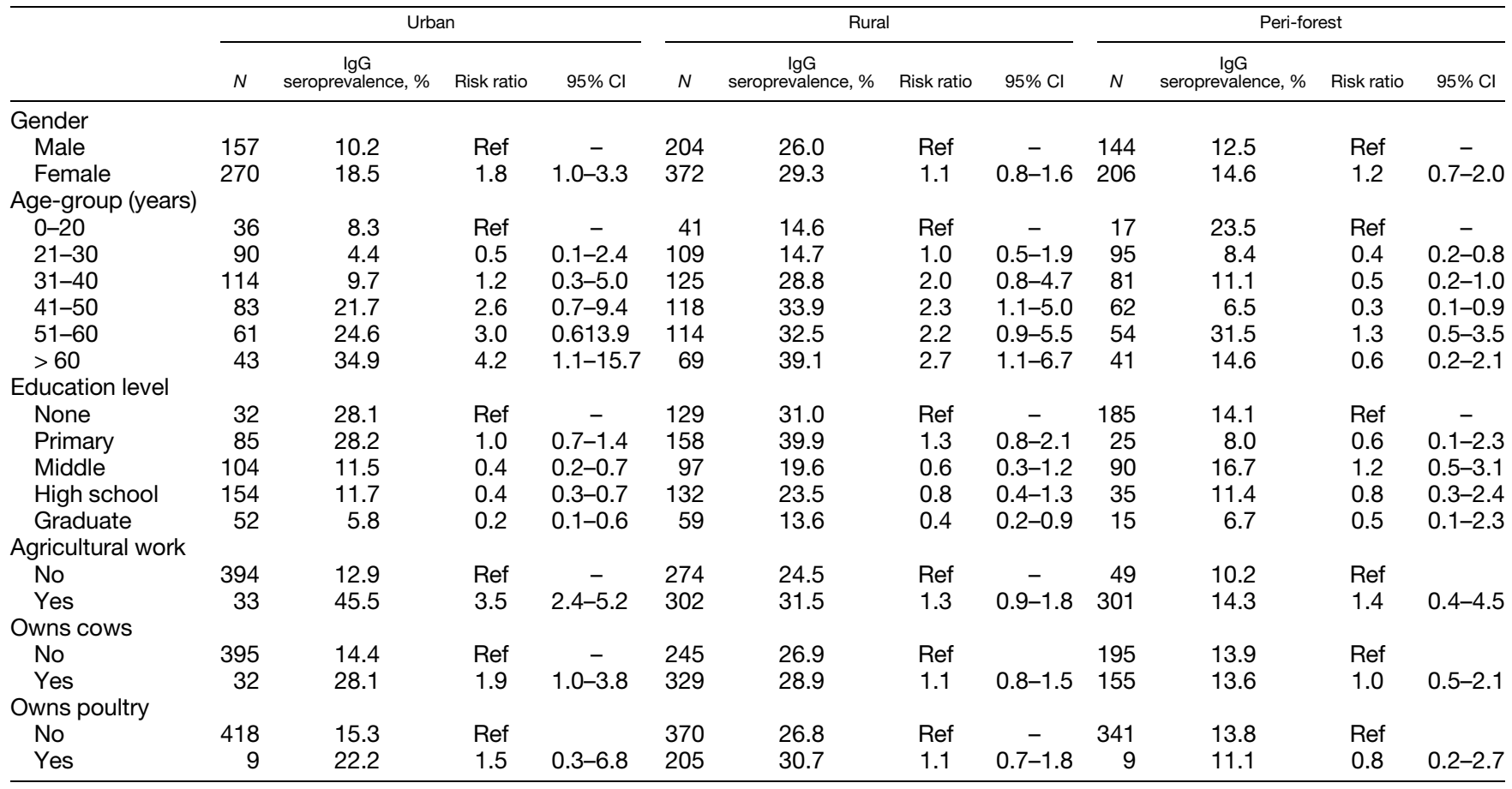

were substantial, then one would expect a higher proportion of participants to be positive for both infections. This finding suggests that cross-reactivity, while of clinical relevance, especially in acute cases, may be of lesser importance in serological surveys using IgG. In the present study, dual seropositivity was slightly more common than expected, especially between scrub typhus and rickettsial infections under study. However, this may also represent true dual

TABLE 3

Murine typhus effect modification by geography

\begin{tabular}{|c|c|c|c|c|c|c|c|c|}
\hline & & Urba & & & & Rura & & \\
\hline & N & IgG seroprevalence, \% & Risk ratio & $95 \% \mathrm{Cl}$ & N & IgG seroprevalence, \% & Risk ratio & $95 \% \mathrm{Cl}$ \\
\hline Gender & & & & & & & & \\
\hline Male & 270 & 5.9 & Ref & - & 372 & 10.5 & Ref & - \\
\hline Female & 157 & 4.5 & 1.3 & $0.4-4.9$ & 204 & 5.4 & 1.9 & $1.0-3.7$ \\
\hline Age-group (yez & & & & & & & & \\
\hline $0-20$ & 36 & 2.8 & Ref & - & 41 & 4.9 & Ref & - \\
\hline $21-30$ & 90 & 5.6 & 2.0 & $0.3-11.5$ & 109 & 5.5 & 1.1 & $0.2-5.7$ \\
\hline $31-40$ & 114 & 4.4 & 1.6 & $0.2-16.3$ & 125 & 12.0 & 2.5 & $0.7-8.1$ \\
\hline $41-50$ & 83 & 9.6 & 3.5 & $0.6-20.6$ & 118 & 6.8 & 1.4 & $0.3-6.2$ \\
\hline $51-60$ & 61 & 1.6 & 0.6 & $0.0-10.0$ & 114 & 11.4 & 2.3 & $0.8-6.6$ \\
\hline$>60$ & 43 & 7.0 & 2.5 & $0.5-12.8$ & 69 & 8.7 & 1.8 & $0.6-5.5$ \\
\hline Education leve & & & & & & & & \\
\hline None & 32 & 3.1 & Ref & - & 129 & 13.2 & Ref & - \\
\hline Primary & 85 & 8.2 & 2.6 & $0.2-28.7$ & 158 & 8.9 & 0.7 & $0.3-1.5$ \\
\hline Middle & 104 & 4.8 & 1.5 & $0.2-11.5$ & 97 & 4.1 & 0.3 & $0.1-0.8$ \\
\hline High school & 154 & 5.2 & 1.7 & $0.2-16.4$ & 132 & 9.1 & 0.7 & $0.3-1.5$ \\
\hline Graduate & 52 & 3.9 & 1.2 & $0.1-15.8$ & 59 & 5.1 & 0.4 & $0.1-1.6$ \\
\hline Lake/pond with & & & & & & & & \\
\hline No & 313 & 2.9 & Ref & - & 414 & 5.8 & Ref & - \\
\hline Yes & 114 & 12.3 & 4.3 & $1.4-13.3$ & 162 & 16.1 & 2.8 & $1.3-5.8$ \\
\hline House yard ha & & & & & & & & \\
\hline No & 31 & 22.6 & Ref & - & 25 & - & Ref & - \\
\hline Yes & 396 & 4.0 & 0.2 & $0.0-0.9$ & 324 & - & 0.4 & $0.2-0.8$ \\
\hline Pet dog & & & & & & & & \\
\hline No & 270 & 2.6 & Ref & - & 331 & 5.4 & Ref & - \\
\hline Yes & 157 & 10.2 & 3.9 & $1.4-10.8$ & 244 & 13.1 & 2.4 & $1.3-4.6$ \\
\hline Owns poultry & & & & & & & & \\
\hline No & 418 & 5.5 & Ref & - & 370 & 7.8 & Ref & - \\
\hline Yes & 9 & 0.0 & - & - & 205 & 10.2 & 1.3 & $0.7-2.4$ \\
\hline
\end{tabular}


TABLE 4

Spotted fever effect modification by geography

\begin{tabular}{|c|c|c|c|c|c|c|c|c|c|c|c|c|}
\hline & \multicolumn{4}{|c|}{ Urban } & \multicolumn{4}{|c|}{ Rural } & \multicolumn{4}{|c|}{ Peri-forest } \\
\hline & N & $\underset{\operatorname{lgG}}{\text { seroprevalence, } \%}$ & Risk ratio & $95 \% \mathrm{Cl}$ & $N$ & $\begin{array}{c}\lg G \\
\text { seroprevalence, } \%\end{array}$ & Risk ratio & $95 \% \mathrm{Cl}$ & N & $\begin{array}{c}\operatorname{lgG} \\
\text { seroprevalence, } \%\end{array}$ & Risk ratio & $95 \% \mathrm{Cl}$ \\
\hline \multicolumn{13}{|l|}{ Gender } \\
\hline Male & 157 & 2.6 & 1 & - & 204 & 12.8 & 1 & - & 144 & 17.4 & 1 & - \\
\hline Female & 270 & 4.4 & 1.7 & $0.5-5.6$ & 372 & 12.6 & 1.0 & $0.7-1.5$ & 206 & 13.1 & 0.8 & $0.4-1.3$ \\
\hline \multicolumn{13}{|c|}{ Age-group (years) } \\
\hline $0-20$ & 36 & 2.8 & Ref & & 41 & 2.4 & Ref & - & 17 & 5.9 & Ref & - \\
\hline $21-30$ & 90 & 3.3 & 1.2 & $0.3-5.3$ & 109 & 11.9 & 4.9 & $0.6-38.0$ & 95 & 14.7 & 2.5 & $0.6-11.4$ \\
\hline $31-40$ & 114 & 5.3 & 1.9 & $0.2-17.0$ & 125 & 11.2 & 4.6 & $0.7-29.3$ & 81 & 12.4 & 2.1 & $0.3-14.3$ \\
\hline $41-50$ & 83 & 1.2 & 0.4 & $0.0-6.4$ & 118 & 17.8 & 7.3 & $1.1-49.5$ & 62 & 21.0 & 3.6 & $0.7-19.5$ \\
\hline $51-60$ & 61 & 6.6 & 2.4 & $0.2-22.4$ & 114 & 14.0 & 5.8 & $0.8-40.3$ & 54 & 14.8 & 2.5 & $0.4-15.3$ \\
\hline$>60$ & 427 & 3.8 & 0.8 & $0.1-12.1$ & 69 & 11.6 & 4.8 & $0.6-41.0$ & 41 & 14.6 & 2.5 & $0.4-15.7$ \\
\hline \multicolumn{13}{|l|}{ Education level } \\
\hline None & 32 & 3.1 & Ref & & 129 & 20.2 & Ref & & 185 & 21.6 & Ref & - \\
\hline Primary & 85 & 8.2 & 2.6 & $0.8-8.2$ & 158 & 16.5 & 0.8 & $0.4-1.6$ & 25 & 4.0 & 0.2 & $0.0-1.4$ \\
\hline Middle & 104 & 2.9 & 0.9 & $0.1-6.4$ & 97 & 11.3 & 0.6 & $0.3-1.2$ & 90 & 7.8 & 0.4 & $0.2-0.7$ \\
\hline High school & 154 & 3.3 & 1.0 & $0.2-6.6$ & 132 & 6.8 & 0.3 & $0.2-0.6$ & 35 & 11.4 & 0.5 & $0.2-1.5$ \\
\hline Graduate & 52 & 0.0 & - & - & 59 & 1.7 & 0.1 & $0.0-0.5$ & 15 & 0.0 & - & - \\
\hline \multicolumn{13}{|c|}{ Agricultural work } \\
\hline No & 394 & 3.6 & 1 & - & 274 & 8.0 & 1 & - & 49 & 4.1 & 1 & - \\
\hline Yes & 33 & 6.1 & 1.7 & $0.3-10.3$ & 302 & 17.0 & 2.1 & $1.4-3.3$ & 301 & 16.6 & 4.1 & $1.6-10.5$ \\
\hline \multicolumn{13}{|c|}{ Forest within $300 \mathrm{~m}$} \\
\hline No & 425 & 3.8 & Ref & - & 428 & 9.6 & Ref & - & 161 & 5.0 & Ref & - \\
\hline Yes & 2 & 0.0 & - & - & 148 & 21.6 & 2.3 & $1.2-4.3$ & 189 & 23.3 & 4.7 & $2.3-9.4$ \\
\hline \multicolumn{13}{|c|}{ Owns vegetable Patch } \\
\hline No & 393 & 4.1 & Ref & - & 133 & 9.8 & Ref & - & 2 & 0.0 & Ref & - \\
\hline Yes & 34 & 0.0 & - & - & 442 & 13.6 & 1.4 & $0.7-2.8$ & 348 & 14.9 & - & - \\
\hline \multicolumn{13}{|c|}{ Collects firewood } \\
\hline No & 424 & 3.3 & Ref & - & 405 & 11.6 & Ref & - & 265 & 12.8 & Ref & - \\
\hline Yes & 3 & 66.7 & 20.2 & $11.9-34.3$ & 171 & 15.2 & 1.3 & $0.8-2.1$ & 85 & 21.2 & 1.7 & $0.9-2.9$ \\
\hline \multicolumn{13}{|c|}{ Cuts grass for fodder } \\
\hline No & 419 & 3.3 & Ref & & 327 & 12.2 & Ref & - & 59 & 5.1 & Ref & - \\
\hline Yes & 8 & 25.0 & 7.5 & $3.1-18.0$ & 249 & 13.2 & 1.1 & $0.6-1.9$ & 291 & 16.8 & 3.3 & $1.4-7.8$ \\
\hline \multicolumn{13}{|l|}{ Owns cows } \\
\hline No & 395 & 3.0 & Ref & - & 245 & 11.8 & Ref & & 195 & 12.8 & Ref & - \\
\hline Yes & 32 & 12.5 & 4.1 & $1.1-15.1$ & 329 & 13.4 & 1.1 & $0.8-1.6$ & 155 & 17.4 & 1.4 & $0.8-2.2$ \\
\hline \multicolumn{13}{|l|}{ Owns goats } \\
\hline No & 414 & 3.6 & Ref & - & 456 & 11.6 & Ref & - & 264 & 10.2 & Ref & - \\
\hline Yes & 13 & 7.7 & 2.1 & $0.4-12.0$ & 119 & 16.8 & 1.4 & $0.9-2.4$ & 85 & 29.4 & 2.9 & $1.7-4.7$ \\
\hline \multicolumn{13}{|l|}{ Owns poultry } \\
\hline No & 418 & 3.6 & Ref & - & 370 & 9.5 & Ref & - & 341 & 15.0 & Ref & - \\
\hline Yes & 9 & 11.1 & 3.1 & $0.3-28.9$ & 205 & 18.5 & 2.0 & $1.1-3.5$ & 9 & 11.1 & 0.7 & $0.2-2.5$ \\
\hline
\end{tabular}

seropositivity due to overlapping risk factors such as agricultural work (scrub typhus and spotted fever) and rural plain geography (scrub typhus and murine typhus). If crossreactivity were a substantial problem, then one would have expected a far higher proportion of dual seropositivity among the three pathogens. Furthermore, if there was relevant crossreactivity between spotted fever and murine typhus antibodies, we should have found some cases of murine typhus seropositivity to occur in hill areas where spotted fever was common.

Finally, the study lacked a formal sampling frame for enrolling villages and individuals within clusters. The aim of the study was to study risk factors for the three pathogens which are easier and more precise to do if the study population is at high risk. We therefore chose to enroll presumed high-risk clusters of Orientia/rickettsial infection. Because of the predominance of scrub typhus in the area, the sample was probably biased toward enrolling scrub typhus clusters, and not necessarily represents high-risk murine typhus and spotted fever clusters, which often may not be diagnosed using reliable tests. The enrollment of peri-forest hill villages was largely performed purposively, because of logistical constraints. Enrollment of participants within households was restricted to those present at the time of the survey, which is likely to bias the sample toward those being at home more often, such as women and the elderly. People commuting to work and those working away in fields may have been underrepresented. Because of the nonrandom enrollment of clusters and individuals, absolute seroprevalence estimates in this study may not be generally applicable to similar settings in South India. There is, however, little reason to assume that the study procedures introduced substantial bias to the risk ratio estimates.

Testing a large number of risk factors as performed here may lead to associations arising by chance. Nevertheless, it seems justified to conclude that in South India, urban, rural plains, and hill settings display distinct epidemiological pattern of Orientia and rickettsial infections. Although scrub typhus and spotted fever were associated with known risk factors in this study, the study suggests a different ecology of murine typhus transmission compared with other studies done in Asia, possibly involving domestic animals. Future studies should 1) explore vector-host relationships especially 
TABLE 5

Multivariable analysis

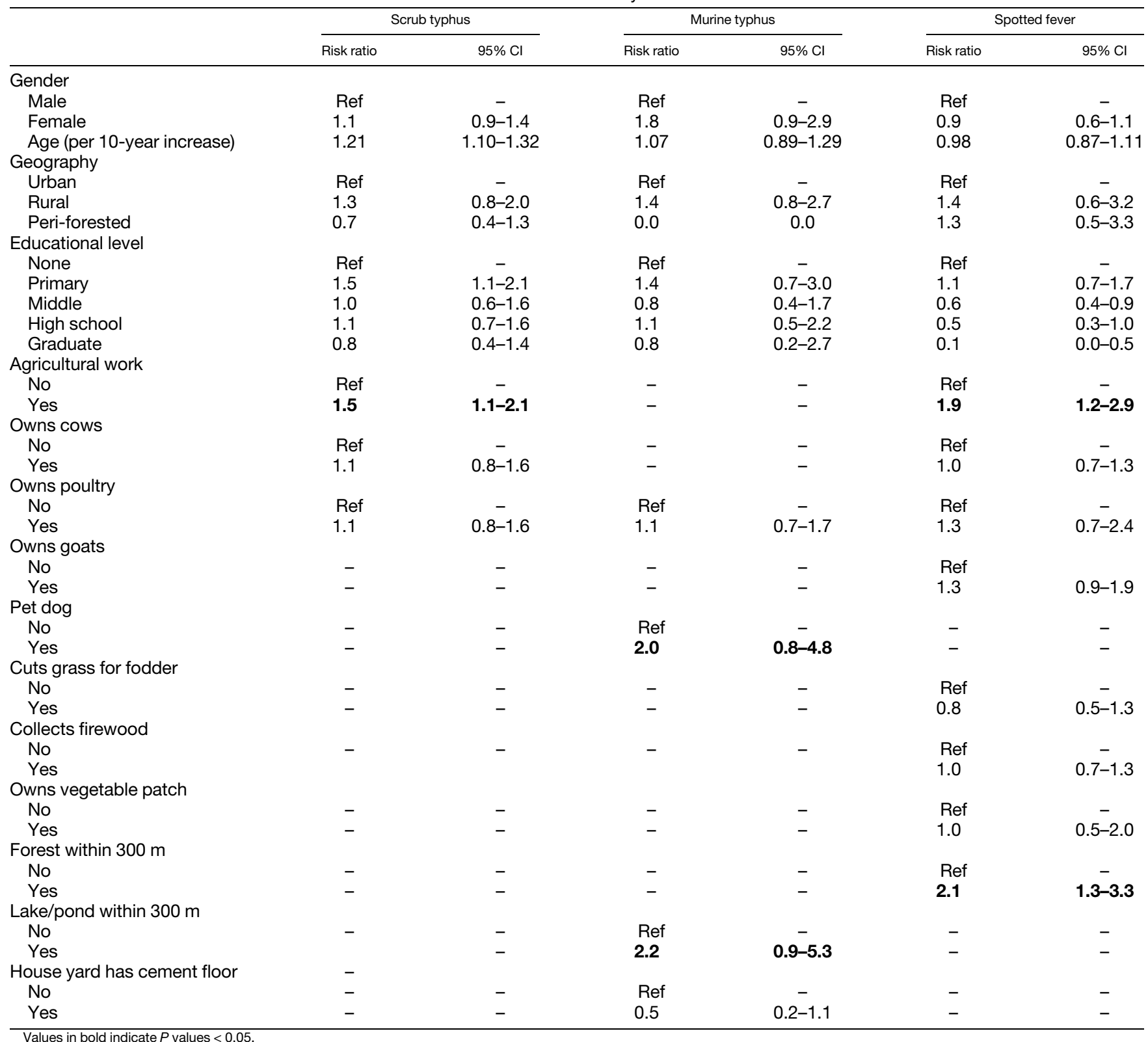

for murine typhus, 2) provide a more complete clinical picture for spotted fever and murine typhus which are rarely diagnosed in India, and 3) determine for all three infections studied here the longevity of $\lg G$ antibodies following infection, which will allow a more meaningful interpretation of seroprevalence data.

Received August 29, 2019. Accepted for publication February 11, 2020.

Published online May 26, 2020.

Acknowledgments: We thank all study participants. We thank R. Ramki and S. Sathiyamoorthi for their help in sample collection.

Financial support: This research work was funded under the Grant-in Aid (GIA) scheme of the Department of Health Research (DHR),
Ministry of Family Health and Welfare, Government of India vide grant No.V 25011/65/2016-GIA-DHR

Authors' addresses: Carol S. Devamani, Department of RUHSA, Christian Medical College, Vellore, India, E-mail: caroldevamani@ hotmail.com. Wolf-Peter Schmidt, Department for Disease Control, London School of Hygiene and Tropical Medicine, London, United Kingdom, E-mail: wolf-peter.schmidt@lshtm.ac.uk. Koya Ariyoshi, Department of Clinical Medicine, Institute of Tropical Medicine, Nagasaki University, Nagasaki, Japan, E-mail: kari@nagasaki-u.ac.jp. Arumugam Anitha, Saravanan Kalaimani, and John A. J. Prakash, Department of Clinical Microbiology, Christian Medical College, Vellore, India, E-mails: anithamalar3108@gmail.com, prakjaj@cmcvellore.ac.in, and prakjaj@cmcvellore.ac.in.

This is an open-access article distributed under the terms of the Creative Commons Attribution (CC-BY) License, which permits unrestricted use, distribution, and reproduction in any medium, provided the original author and source are credited. 


\section{REFERENCES}

1. Tamura A, Ohashi N, Urakami H, Miyamura S, 1995. Classification of Rickettsia tsutsugamushi in a new genus, Orientia gen. nov., as Orientia tsutsugamushi comb. nov. Int J Syst Bacteriol 45: 589-591.

2. Paris DH, Shelite TR, Day NP, Walker DH, 2013. Unresolved problems related to scrub typhus: a seriously neglected lifethreatening disease. Am J Trop Med Hyg 89: 301-307.

3. Parola $\mathrm{P}$ et al., 2013. Update on tick-borne rickettsioses around the world: a geographic approach. Clin Microbiol Rev 26: 657-702.

4. Nogueras MM, Pons I, Pla J, Ortuño A, Miret J, Sanfeliu I, Segura $F, 2013$. The role of dogs in the eco-epidemiology of Rickettsia typhi, etiological agent of murine typhus. Vet Microbiol 163: 97-102.

5. Azad AF, 1990. Epidemiology of murine typhus. Annu Rev Entomol 35: 553-569.

6. Kelly DJ, Fuerst PA, Ching WM, Richards AL, 2009. Scrub typhus: the geographic distribution of phenotypic and genotypic variants of Orientia tsutsugamushi. Clin Infect Dis 48 (Supp/ 3): S203-S230.

7. Weitzel T, Dittrich S, Lopez J, Phuklia W, Martinez-Valdebenito C, Velásquez K, Blacksell SD, Paris DH, Abarca K, 2016. Endemic scrub typhus in South America. N Engl J Med 375: 954-961.

8. Maina AN, Farris CM, Odhiambo A, Jiang J, Laktabai J, Armstrong J, Holland T, Richards AL, O'Meara WP, 2016. Q fever, scrub typhus, and rickettsial diseases in children, Kenya, 2011-2012. Emerg Infect Dis 22: 883-886.

9. Rathi N, Rathi A, 2010. Rickettsial infections: Indian perspective. Indian Pediatr 47: 157-164.

10. Gopinath KG, Chrispal A, Boorugu H, Chandy S, Prakash JJ, Abraham AM, Abraham OC, Thomas K, 2014. Clinicoepidemiological profile of seven adults with spotted fever from a tertiary care hospital in south India. Trop Doct 44: 89-91.

11. George T, Rajan SJ, Peter JV, Hansdak SG, Prakash JAJ, lyyadurai R, Mathuram A, Antonisamy B, Ramanathan K, Sudarsanam TD, 2018. Risk factors for acquiring scrub typhus among the adults. $J$ Glob Infect Dis 10: 147-151.

12. Varghese GM, Raj D, Francis MR, Sarkar R, Trowbridge $P$, Muliyil $\mathrm{J}, 2016$. Epidemiology \& risk factors of scrub typhus in south India. Indian J Med Res 144: 76-81.

13. Trowbridge P, Divya P., Premkumar PS, Varghese GM, 2017. Prevalence and risk factors for scrub typhus in south India. Trop Med Int Health 22: 576-582.

14. Devamani CS, Prakash JAJ, Alexander N, Suzuki M, Schmidt WP, 2019. Hospitalisations and outpatient visits for undifferentiated fever attributable to scrub typhus in rural south India: retrospective cohort and nested case-control study. PLoS Negl Trop Dis 13: e0007160.

15. Vallee J, Thaojaikong T, Moore CE, Phetsouvanh R, Richards AL, Souris M, Fournet F, Salem G, Gonzalez JP, Newton PN, 2010. Contrasting spatial distribution and risk factors for past infection with scrub typhus and murine typhus in Vientiane city, Lao PDR. PLoS Negl Trop Dis 4: e909.

16. Leithwaite RSS, 1936. The typhus group of diseases in Malaya VIII. The relation of the tsutsugamushi disease (including rural typhus) to urban typhus. B J Exp Path 17: 461-466.

17. Traub R, Wisseman CL, 1978. The ecology of murine typhus-a critical review. Trop Dis Bull 75: 237-317.

18. Dennis DT, Hadi TR, Brown RJ, Sukaeri S, Leksana B, Cholid R, 1981. A survey of scrub and murine typhus in the Ancol section of Jakarta, Indonesia. Southeast Asian J Trop Med Public Health 12: 574-580.

19. Richards AL et al., 1997. Seroepidemiologic evidence for murine and scrub typhus in Malang, Indonesia. Am J Trop Med Hy 57: 91-95.

20. Tay ST, Kamalanathan M, Rohani MY, 2003. Antibody prevalence of Orientia tsutsugamushi, Rickettsia typhi and TT118 spotted fever group rickettsiae among Malaysian blood donors and febrile patients in the urban areas. Southeast Asian J Trop Med Public Health 34: 165-170.

21. Zhang LJ, Li XM, Zhang DR, Zhang JS, Di Y, Luan MC, Fu XP, 2007. Molecular epidemic survey on co-prevalence of scrub typhus and marine typhus in Yuxi city, Yunnan province of China. Chin Med J 120: 1314-1318.

22. Hamaguchi S et al., 2015. Clinical and epidemiological characteristics of scrub typhus and murine typhus among hospitalized patients with acute undifferentiated fever in northern Vietnam. Am JTrop Med Hyg 92: 972-978.

23. Park SW, Ha NY, Ryu B, Bang JH, Song H, Kim Y, Kim G, Oh MD, Cho NH, Lee JK, 2015. Urbanization of scrub typhus disease in South Korea. PLoS Negl Trop Dis 9: e0003814.

24. Tshokey T, Stenos J, Durrheim DN, Eastwood K, Nguyen C, Graves SR, 2017. Seroprevalence of rickettsial infections and Q fever in Bhutan. PLoS Negl Trop Dis 11: e0006107.

25. Rose W, Kang G, Verghese VP, Candassamy S, Samuel P, Prakash JJA, Muliyil J, 2019. Risk factors for acquisition of scrub typhus in children admitted to a tertiary centre and its surrounding districts in south India: a case control study. BMC Infect Dis 19: 665.

26. Noden BH, Davidson S, Smith JL, Williams F, 2017. First detection of Rickettsia typhi and Rickettsia felis in fleas collected from client-owned companion animals in the southern great plains. J Med Entomol 54: 1093-1097.

27. Lledo L, Gegundez MI, Serrano JL, Saz JV, Beltran M, 2003. A sero-epidemiological study of Rickettsia typhi infection in dogs from Soria province, central Spain. Ann Trop Med Parasitol 97: 861-864.

28. Bharti AR et al., 2003. Leptospirosis: a zoonotic disease of global importance. Lancet Infect Dis 3: 757-771.

29. Dincy PC, Susanne PA, Leni G, T. S, Meera T, Aj PJ, 2018; Clinicopathological study on rickettsial spotted fever from south India. Trop Doct 48: 325-329.

30. Kalal BS, Puranik P, Nagaraj S, Rego S, Shet A, 2016. Scrub typhus and spotted fever among hospitalised children in south India: clinical profile and serological epidemiology. Indian J Med Microbiol 34: 293-298.

31. Stephen S, Ambroise S, Gunasekaran D, Hanifah M, Sangeetha B, Pradeep J, Sarangapani K, 2018. Serological evidence of spotted fever group rickettsiosis in and around Puducherry, south India-a three years study. J Vector Borne Dis 55: 144-150.

32. Prakash JA, Sohan Lal T, Rosemol V, Verghese VP, Pulimood SA, Reller M, Dumler JS, 2012. Molecular detection and analysis of spotted fever group Rickettsia in patients with fever and rash at a tertiary care centre in Tamil Nadu, India. Pathog Glob Health 106: $40-45$.

33. Podsiadly E, Chmielewski T, Karbowiak G, Kedra E, TylewskaWierzbanowska S, 2011. The occurrence of spotted fever rickettsioses and other tick-borne infections in forest workers in Poland. Vector Borne Zoonotic Dis 11: 985-989.

34. Aung AK, Spelman DW, Murray RJ, Graves S, 2014. Rickettsial infections in southeast Asia: implications for local populace and febrile returned travelers. Am J Trop Med Hyg 91: 451-460.

35. Tay ST, Ho TM, Rohani MY, Devi S, 2000. Antibodies to Orientia tsutsugamushi, Rickettsia typhi and spotted fever group rickettsiae among febrile patients in rural areas of Malaysia. Trans $R$ Soc Trop Med Hyg 94: 280-284.

36. Tay ST, Rohani MY, Ho TM, Devi S, 2002. Isolation and PCR detection of rickettsiae from clinical and rodent samples in Malaysia. Southeast Asian J Trop Med Public Health 33: 772-779.

37. Suttinont $C$ et al., 2006. Causes of acute, undifferentiated, febrile illness in rural Thailand: results of a prospective observational study. Ann Trop Med Parasitol 100: 363-370.

38. Richards AL, Ratiwayanto S, Rahardjo E, Kelly DJ, Dasch GA, Fryauff DJ, Bangs MJ, 2003. Serologic evidence of infection with ehrlichiae and spotted fever group rickettsiae among residents of Gag Island, Indonesia. Am J Trop Med Hyg 68: 480-484.

39. Brown GW, Robinson DM, Huxsoll DL, Ng TS, Lim KJ, 1976. Scrub typhus: a common cause of illness in indigenous populations. Trans $R$ Soc Trop Med Hyg 70: 444-448.

40. Ogrzewalska M, Saraiva DG, Moraes-Filho J, Martins TF, Costa FB, Pinter A, Labruna MB, 2012. Epidemiology of Brazilian spotted fever in the atlantic forest, state of Sao Paulo, Brazil. Parasitology 139: 1283-1300. 
41. de Oliveira SV et al., 2016. An update on the epidemiological situation of spotted fever in Brazil. J Venom Anim Toxins Incl Trop Dis 22: 22

42. Varghese GM, Rajagopal VM, Trowbridge P, Purushothaman D, Martin SJ, 2018. Kinetics of IgM and IgG antibodies after scrub typhus infection and the clinical implications. Int $J$ Infect Dis 71: $53-55$

43. Sando E, Ariyoshi K, Fujita H, 2018. Serological cross-reactivity among Orientia tsutsugamushi serotypes but not with Rickettsia japonica in Japan. Trop Med Infect Dis 3: E74. 